\title{
28 Research Soure \\ ARID1A Upregulation Predicts Poor Survival in Patients With Liver Cancer
}

\section{Feng Jiang \\ Jiangsu Province Hospital \\ Ke Wei \\ Jiangsu Province Hospital \\ Ming Wang \\ Jiangsu Province Hospital}

Chuyan Wu ( $\nabla$ chuyan_w@hotmail.com )

Department of Rehabilitation Medicine, the First Affiliated Hospital of Nanjing Medical University, Nanjing 210000, China

\section{Research Article}

Keywords: Gene Expression, Kaplan-Meier Estimate, Liver Neoplasm, Survival Analysis

Posted Date: January 19th, 2021

DOl: https://doi.org/10.21203/rs.3.rs-146505/v1

License: (1) (1) This work is licensed under a Creative Commons Attribution 4.0 International License. Read Full License 


\section{Abstract}

Objective: ARID1A has been identified as a possible biomarker for certain cancers. There is, however, some debate regarding its function in liver cancer.

Methods: Associations between clinical variables and ARID1A were evaluated. Cox and Kaplan - Meier analysis were used to examine clinical pathological factors linked to overall survival of patients with liver cancer. Gene set enrichment analysis (GSEA) was conducted using the dataset of the cancer genome atlas(TCGA).

Results: High expression of ARID1A was correlated with the gender and tumor topography (T) diagnosis of liver cancer. Patients with elevated ARID1A expression had poorer prognosis than those with low ARID1A expression. The study also showed that ARID1A was an independent risk factor for overall survival. GSEA established pathways involved in ERBB signaling, cancer, insulin signaling, mTOR signaling, MAPK signaling, VEGF signaling, Ubiquitin signaling, and Wnt signaling as differentially enriched in ARID1A-high expression liver cancer.

Conclusion: ARID1A has been shown to be expressed at high rates of liver cancer and to represent a possible independent molecular marker for diagnosis and prognosis of liver cancer.

\section{Introduction}

Hepatic carcinomas (HCC), composite hepatocellular carcinoma and cholangiocarcinoma(HCC-CC), as well as other hepatic malignancies (Other) have become the second leading source and the sixth most common malignant source of cancer-related death[1]. Globally, the incidence rate of liver cancer is very high, and there is an increasing trend every year. On average, the total rate of survival over five years is below $5 \%[2]$.

At present, there are very few early detection methods for liver cancer[3]. There have been many mutations genes in liver cancer in the previous researches, among which ARID1A is the most controversial one[4]. Functional studies have shown that ARID1A influences cell proliferation and metastases of cancer[5].The colony for mation in soft agar has been specifically inhibited in the ARID1Areexpressed breast cancer cell line [6]. The absence of ARID1A in gynecological cancer cells enhanced the cell proliferation and the development of the colony[7]. In fact, silencing ARID1A enhanced liver cancer cell infiltration and migration. But other findings point out a more complex function for ARID1A in tumorigenesis and in some cases some of the SWI / SNF components are oncogenic[8,9]. All these studies indicate that ARID1A may play a role in tumor initiation, especially in the promotion of liver cancer.

This work aimed to examine the diagnostic and prognostic importance of ARID1A expression in liver cancer using the Cancer genome atlas (TCGA) database. And we used Gene set enrichment analysis 
(GSEA) to further examine the biological pathways involved in the pathogenesis-associated ARID1A regulatory networks for liver cancer.

\section{Materials And Methods}

\section{Data collection}

TCGA database was selected to predict the expression lever of ARID1A in liver cancer and normal liver tissues. In TCGA database (http://cancergenome.nih.gov/), a total of 407 cases of cancer and 58 cases of normal liver tissues containing ARID1A mRNA expression information were downloaded. R3.6.1 software was performed to analyze the differential expression levels of ARID1A mRNA between the two groups.

\section{Gene set enrichment analysis}

GSEA is a computational approach to detecting whether a set of predefined genes displays statistically significant differential expression between high and low expression groups[10,11]. Datasets and label phenotype files have been created and uploaded to GSEA software. We use ARID1A-low and ARID1A-high phenotype labels. Gene set permutations for each analysis were performed 1000-times. And the gene sets the usual P-value.

\section{Statistical analysis}

The relations between clinical and ARID1A expression were tested through logistic regression and Kolmogorov-Smirnov analysis. The kaplan meierand cox regression method was used to classify therapeutic variables that were associated with complete survival of patients infected with liver cancer. Then we used multivariate cox analysis to examine ARID1A expression's function in survival along with those clinical traits (age, gender, grade, tumor topography $(T)$, distant metastasis status $(M)$, and lymph node status $(\mathrm{N})$ ). Low and high ARID1A expression was found out based on the median values. Median risk score of ARID1A expression was used as the cut off value, all these patients were classified as high expression or low expression groups. And R software (v3.6.1) was used for all statistical analysis.

\section{Results}

\section{High expression of ARID1A in liver cancer}

TCGA information was analyzed by bioinformatics approach to look through ARID1A mRNA's differential expression levels between normal tissues and liver cancer. Scatter plot and paired difference plot were used respectively. As shown in Figure A (a) and Figure A (b), ARID1A mRNA level was remarkably higher in liver cancer than normal liver tissues (All $p$ values< 0.05 ).

\section{Associations between clinical parameters and ARID1A expression}


Clinical information that pertained to 418 liver cancer patients from the TCGA were analyzed, and involved the patient's gender, age, clinical stage, histological grade, tumor topography, distance metastasis, and lymph node (TMN) categorization, survival time, and survival status. As exhibited in Figure B (a-g), ARID1A expression was notably connected with gender $(p=0.008)$, clinical step $(p=0.077)$, Tumor topography classification $(p=0.087)$ and lymph node classification $(p=0.04)$. ARID1A's high expression in liver cancer was associated with gender ( $\mathrm{OR}=0.37$ for male vs. female) significantly and $T$ categorization (OR=1.91 for T3 vs. T1) (Table1).

\section{Survival outcomes and multivariate analysis}

As illustrated in Figure C, ARID1A's excellent expression was connected with poor whole survival $(P=0.038)$ (Figure $C)$. The univariate analysis showed that excellent ARID1A suggested that high ARID1A expression was considerably associated adverse over-all survival [hazard ratio (HR): $1.0985 ; 95 \% \mathrm{Cl}$ : 1.0341-1.1669; $P=0.0023$, Table2]. Stage, $T$ and $M$ classification (Table2) were included by those clinical factors that were connected with poor survival. The multivariate analysis included all of the variables. And multivariate cox analysis disclosed that excellent ARID1A expression remained a self-governing risk factor for whole survival with a HR of $1.0682(95 \% \mathrm{Cl}$ : 1.0004-1.1406, $\mathrm{P}=0.0487$, Figure $\mathrm{D}$ and Table2), as well as T classification ( $\mathrm{HR}=2.1529,95 \% \mathrm{Cl}: \mathrm{P}=0.0499,1.0004-4.6332$, Table2) among liver cancer patients.

\section{GSEA identifies ARID1A-associatedsignaling pathways}

ARID1A-associated signal pathways to screen for possible signaling pathways that are differentially activated in liver cancer are identified by GSEA. GSEA was performed by comparing the down and up ARID1A expression datasets. The nominal P-value sets $<0.05$ and FDR q-value $<0.25$ is found to be significantly enriched. Significant differences were revealed by GSEA in the MSigDB collection's enrichment (h.all.v6.2.symbols.gmt). According to their standardized enrichment marks, the most considerably enriched signaling pathways were identified. ERBB signaling, cancer signaling, insulin signaling, mTOR signaling, MAPK signaling, VEGF signaling, Wnt signaling, and Ubiquitin signaling were differentially associated with ARID1A high expression phenotype, differentially correlated with low expression phenotype ARID1A is Parkinsons disease and Oxidative signalling, as illustrated in Figure D.

\section{Discussion}

The analysis of TCGA information in the present research showed that ARID1A is over expressed in liver cancer. Previous work has shown that ARID1A plays a major role as a tumor suppressor and its expression levels have been strongly correlated with prognosis for tumor patients, including gastric, breast cancer, prostate and pulmonary cancer.[12,13]. However, Zhao etal have demonstrated that $83 \%$ of tumors show over expression of ARID1A compared with adjacent tissues of liver cancer [14]. We contrasted two forms of plots between the regular group and the experimental group. The first graph was the separation map and the second graph was the pairing discrepancy map that indicated that there was a substantial gap between the two classes. These findings indicate that ARID1A is already strongly 
expressed in liver cancer from a bioinformatic research viewpoint and may play a part in the initiation of tumors.

And we also tested its associations with the clinical and predicted properties of patients, and its occurrence was substantially linked to gender $(P<0.05)$, lymph node stage $(N)$ classification $(P<0.05)$. The incidence rate of male and female liver cancer is 3-5:1[15], and our results also show gender differences. In our study, more male patients than female were included in and ARID1A expression is higher in female patients. Some studies suggest that ARID1A will be lost in the later stage of the development of liver cancer $[16,17,18]$. Our study also shows that although the expression in N1 phase is higher than that in N0 phase, the expression in T4 phase suddenly decreased, even lower than that in T1 phase. All these results suggest that ARID1A might play a key role in liver cancer [4].

Patients whose liver cancer tissues showed ARID1A's high expression owned a short prognosis. Throughout the later stage of the survival graph, the high-expression and low-expression community crossed the graph, which could be triggered by ARID1A mutation or failure in the later stage of liver cancer, suggesting also that ARID1A may play complicated roles in the production and incidence of liver cancer. An earlier research has reported that ARID1A failure has been shown to be linked to tumor size, but has no prognostic value in HCC patients[14,19]. ARID1A that was promoted cancer initiation was regarded partly by using its transcriptional restriction of Cyp2e1 expression, which in turn aroused reactive oxygen species(ROS) output from endogenous metabolites [4]. MultiCox analysis and UniCox analysis showed that ARID1A expression plays a considerable function in whole survival and maybe can be used as a clinically helpful self-governing prognostic factor for liver cancer.

To further evaluate ARID1A's roles in liver cancer, GSEA was performed using TCGA data. GSEA indicated that genes that were complicated in ERBB signaling, cancer, insulin signaling, mTOR signaling, MAPK signaling, VEGF signaling, Ubiquitin signaling, and Wnt signaling were associated with the ARID1A excellent expression phenotype differentially. ARID1Alow expression phenotype mainly related to Parkinsons_disease signaling and Oxidative signaling. MAPK and VEGF signal pathways play a crucial function in the colonization and metastasization of liver cancer[20]. PI3K / Akt / mTOR and PKB / Akt signaling pathways play an significant function in the initiation, development and prognosis of liver cancer[21]. Wnt signaling pathway affects the occurrence and development of liver cancer by activating its downstream target genes[22,23,24]. This indicates that ARID1A may serve as a potential prognostic marker for hepatic cancer pronostics and diagnoses.

To our knowledge, this is the first work to find a correlation between survival and ARID1A expression and report that ARID1A may provide self-governing pronostic factors to minimize liver cancer clinical parameters using TCGA database that degrades liver cancer. Although we have shown that ARID1A expression is a potential self-governing molecular marker for liver cancer diagnosis and prognosis, some limitations of our work are still to be noted. Our results were focused primarily on bioinformatic research. Therefore, further more experimental validation should to be implemented to confirm biological importance of ARID1Aexpression in liver cancer. 


\section{Conclusions}

The study that prevails indicates that ARID1A is active in malignancy and tumorigenesis of liver cancer and therefore can be used in patients with liver cancer as an alternative diagnostic and/or prognostic biomarker.

\section{Declarations}

\section{Consent for publication}

All authors consent to the publication of the manuscript.

\section{Availability of data and materials}

The datasets used during the present study are available from TCGA

(https://portal.gdc.cancer.gov/repository) database.

\section{Competing interest}

The authors declare that there is no conflict of interest.

\section{Authors' contributions}

CY.W designed the study; K.W and M.W collected the data; F.J and CY.W did the statistic analysis; F.J prepared the manuscript draft; CY.W revised the manuscript; all of the authors approved the final manuscript.

\section{Acknowledgements}

Not applicable.

\section{Funding}

Chuyan Wu was supported by the China Scholarship Council for 1 y study at the University of the Johns Hopkins.

\section{References}

[1] L.A. Torre, F. Bray, R.L. Siegel, J. Ferlay, J. Lortet-Tieulent, A. Jemal, Global cancer statistics, 2012, CA Cancer J Clin 65 (2015) 87-108.

[2] M.I. Shariff, I.J. Cox, A.I. Gomaa, S.A. Khan, W. Gedroyc, S.D. Taylor-Robinson, Hepatocellular carcinoma: current trends in worldwide epidemiology, risk factors, diagnosis and therapeutics, Expert Rev Gastroenterol Hepatol 3 (2009) 353-367.

[3] Y. Fu, X. Xu, D. Huang, D. Cui, L. Liu, J. Liu, Z. He, J. Liu, S. Zheng, Y. Luo, Plasma Heat Shock Protein 90alpha as a Biomarker for the Diagnosis of Liver Cancer: An Official, Large-scale, and Multicenter 
Clinical Trial, EBioMedicine 24 (2017) 56-63.

[4] X. Sun, S.C. Wang, Y. Wei, X. Luo, Y. Jia, L. Li, P. Gopal, M. Zhu, I. Nassour, J.C. Chuang, T. Maples, C. Celen, L.H. Nguyen, L. Wu, S. Fu, W. Li, L. Hui, F. Tian, Y. Ji, S. Zhang, M. Sorouri, T.H. Hwang, L. Letzig, L. James, Z. Wang, A.C. Yopp, A.G. Singal, H. Zhu, Arid1a Has Context-Dependent Oncogenic and Tumor Suppressor Functions in Liver Cancer, Cancer Cell 32 (2017) 574-589 e576.

[5] Q. Cao, C. Wang, Y. Ding, D. Xu, S. Qian, H. Shen, J. Qi, ARID1A upregulation predicts better survival in patients with urothelial bladder carcinoma, J Int Med Res (2019) 300060519895687.

[6] A. Mamo, L. Cavallone, S. Tuzmen, C. Chabot, C. Ferrario, S. Hassan, H. Edgren, O. Kallioniemi, O. Aleynikova, E. Przybytkowski, K. Malcolm, S. Mousses, P.N. Tonin, M. Basik, An integrated genomic approach identifies ARID1A as a candidate tumor-suppressor gene in breast cancer, Oncogene 31 (2012) 2090-2100.

[7] D.D. Wang, Y.B. Chen, K. Pan, W. Wang, S.P. Chen, J.G. Chen, J.J. Zhao, L. Lv, Q.Z. Pan, Y.Q. Li, Q.J. Wang, L.X. Huang, M.L. Ke, J. He, J.C. Xia, Decreased expression of the ARID1A gene is associated with poor prognosis in primary gastric cancer, PLoS One 7 (2012) e40364.

[8] C. Kadoch, G.R. Crabtree, Reversible disruption of mSWI/SNF (BAF) complexes by the SS18-SSX oncogenic fusion in synovial sarcoma, Cell 153 (2013) 71-85.

[9] L. Jubierre, A. Soriano, L. Planells-Ferrer, L. Paris-Coderch, S.P. Tenbaum, O.A. Romero, R.S. Moubarak, A. Almazan-Moga, C. Molist, J. Roma, S. Navarro, R. Noguera, M. Sanchez-Cespedes, J.X. Comella, H.G. Palmer, J. Sanchez de Toledo, S. Gallego, M.F. Segura, BRG1/SMARCA4 is essential for neuroblastoma cell viability through modulation of cell death and survival pathways, Oncogene 35 (2016) 5179-5190.

[10] A. Subramanian, P. Tamayo, V.K. Mootha, S. Mukherjee, B.L. Ebert, M.A. Gillette, A. Paulovich, S.L. Pomeroy, T.R. Golub, E.S. Lander, J.P. Mesirov, Gene set enrichment analysis: a knowledge-based approach for interpreting genome-wide expression profiles, Proc Natl Acad Sci U S A 102 (2005) 1554515550.

[11] W. Li, F. Han, M. Fu, Z. Wang, High expression of VCAN is an independent predictor of poor prognosis in gastric cancer, J Int Med Res 48 (2020) 300060519891271.

[12] J.N. Wu, C.W. Roberts, ARID1A mutations in cancer: another epigenetic tumor suppressor?, Cancer Discov 3 (2013) 35-43.

[13] A.F. Hohmann, C.R. Vakoc, A rationale to target the SWI/SNF complex for cancer therapy, Trends Genet 30 (2014) 356-363.

[14] J. Zhao, J. Chen, H. Lin, R. Jin, J. Liu, X. Liu, N. Meng, X. Cai, The Clinicopathologic Significance of BAF250a (ARID1A) Expression in Hepatocellular Carcinoma, Pathol Oncol Res 22 (2016) 453-459. 
[15] A. Recio-Boiles, A. Waheed, H.M. Babiker, Cancer, Liver, StatPearls, Treasure Island (FL), 2020.

[16] J. Huang, Q. Deng, Q. Wang, K.Y. Li, J.H. Dai, N. Li, Z.D. Zhu, B. Zhou, X.Y. Liu, R.F. Liu, Q.L. Fei, H. Chen, B. Cai, B. Zhou, H.S. Xiao, L.X. Qin, Z.G. Han, Exome sequencing of hepatitis B virus-associated hepatocellular carcinoma, Nat Genet 44 (2012) 1117-1121.

[17] F. He, J. Li, J. Xu, S. Zhang, Y. Xu, W. Zhao, Z. Yin, X. Wang, Decreased expression of ARID1A associates with poor prognosis and promotes metastases of hepatocellular carcinoma, $\mathrm{J}$ Exp Clin Cancer Res 34 (2015) 47.

[18] Y. Zhai, R. Kuick, C. Tipton, R. Wu, M. Sessine, Z. Wang, S.J. Baker, E.R. Fearon, K.R. Cho, Arid1a inactivation in an Apc- and Pten-defective mouse ovarian cancer model enhances epithelial differentiation and prolongs survival, J Pathol 238 (2016) 21-30.

[19] M. Uhlen, C. Zhang, S. Lee, E. Sjostedt, L. Fagerberg, G. Bidkhori, R. Benfeitas, M. Arif, Z. Liu, F. Edfors, K. Sanli, K. von Feilitzen, P. Oksvold, E. Lundberg, S. Hober, P. Nilsson, J. Mattsson, J.M. Schwenk, H. Brunnstrom, B. Glimelius, T. Sjoblom, P.H. Edqvist, D. Djureinovic, P. Micke, C. Lindskog, A. Mardinoglu, F. Ponten, A pathology atlas of the human cancer transcriptome, Science 357 (2017).

[20] Y. Tsuboi, T. Ichida, S. Sugitani, T. Genda, J. Inayoshi, M. Takamura, Y. Matsuda, M. Nomoto, Y. Aoyagi, Overexpression of extracellular signal-regulated protein kinase and its correlation with proliferation in human hepatocellular carcinoma, Liver Int 24 (2004) 432-436.

[21] F. Sahin, R. Kannangai, O. Adegbola, J. Wang, G. Su, M. Torbenson, mTOR and P70 S6 kinase expression in primary liver neoplasms, Clin Cancer Res 10 (2004) 8421-8425.

[22] T. Suzuki, H. Yano, Y. Nakashima, O. Nakashima, M. Kojiro, Beta-catenin expression in hepatocellular carcinoma: a possible participation of beta-catenin in the dedifferentiation process, $\mathrm{J}$ Gastroenterol Hepatol 17 (2002) 994-1000.

[23] S. Loeppen, C. Koehle, A. Buchmann, M. Schwarz, A beta-catenin-dependent pathway regulates expression of cytochrome P450 isoforms in mouse liver tumors, Carcinogenesis 26 (2005) 239-248.

[24] L.T. Tien, M. Ito, M. Nakao, D. Niino, M. Serik, M. Nakashima, C.Y. Wen, H. Yatsuhashi, H. Ishibashi, Expression of beta-catenin in hepatocellular carcinoma, World J Gastroenterol 11 (2005) 2398-2401.

\section{Tables}

Table1. Logistic regression of ARID1A expression and clinical pathological characteristics. 


\begin{tabular}{|llll|}
\hline Clinical characteristics & Total & Odds rate in ARDI2 expression & p-Value \\
\hline age(<55 VS >=55) & 370 & $1.19(0.77-1.85)$ & 0.434 \\
\hline gender(male VS female) & 404 & $0.37(0.24-0.57)$ & $<0.001$ \\
\hline grade(G2 VS G1) & 232 & $1.34(0.73-2.50)$ & 0.342 \\
\hline grade(G3 VS G1) & 177 & $1.69(0.89-3.25)$ & 0.108 \\
\hline grade(G4 VS G1) & 67 & $1.39(0.49-1.97)$ & 0.605 \\
\hline stage(2v1) & 284 & $1.03(0.63-1.69)$ & 0.898 \\
\hline stage(3v1) & 275 & $1.67(1.00-2.82)$ & 0.051 \\
\hline stage(4v1) & 199 & $1.72(0.48-6.92)$ & 0.412 \\
\hline T(T2 VS T1) & 303 & $1.26(0.78-2.03)$ & 0.343 \\
\hline T(T3 VS T1) & 284 & $1.91(1.14-3.22)$ & 0.014 \\
\hline T(T4 VS T1) & 212 & $0.76(0.22-2.35)$ & 0.636 \\
\hline M(M1 VS M0) & 311 & $1.34(0.29-6.91)$ & 0.703 \\
\hline N(N1 VS N0) & 298 & $3.11(0.70-21.48)$ & 0.169 \\
\hline
\end{tabular}

Table2. Univariate and multivariate analysis of the relationship between ARID1A expression and overall survival among liver cancer patients.

\begin{tabular}{|llllllllll|}
\hline id & uniCox & \multicolumn{7}{c|}{ multiCox } \\
\cline { 2 - 9 } & HR & HR.95L & HR.95H & pvalue & HR & HR.95L & HR.95H & pvalue \\
\hline age & 1.0050 & 0.9869 & 1.0235 & 0.5912 & 1.0095 & 0.9904 & 1.0290 & 0.3310 \\
\hline gender & 0.7801 & 0.4872 & 1.2492 & 0.3013 & 1.0806 & 0.6417 & 1.8196 & 0.7706 \\
\hline grade & 1.0172 & 0.7459 & 1.3871 & 0.9143 & 1.1027 & 0.7933 & 1.5327 & 0.5608 \\
\hline stage & 1.4172 & 1.2349 & 1.6265 & 0.0000 & 0.7728 & 0.3338 & 1.7894 & 0.5474 \\
\hline T & 1.8044 & 1.4341 & 2.2702 & 0.0000 & 2.1529 & 1.0004 & 4.6332 & 0.0499 \\
\hline M & 3.8498 & 1.2068 & 12.2813 & 0.0228 & 3.8978 & 0.1478 & 102.7721 & 0.4151 \\
\hline N & 2.0218 & 0.4939 & 8.2761 & 0.3276 & 2.5713 & 0.4881 & 13.5439 & 0.2653 \\
\hline ARID1A & 1.0985 & 1.0341 & 1.1669 & 0.0023 & 1.0682 & 1.0004 & 1.1406 & 0.0487 \\
\hline
\end{tabular}


Figures
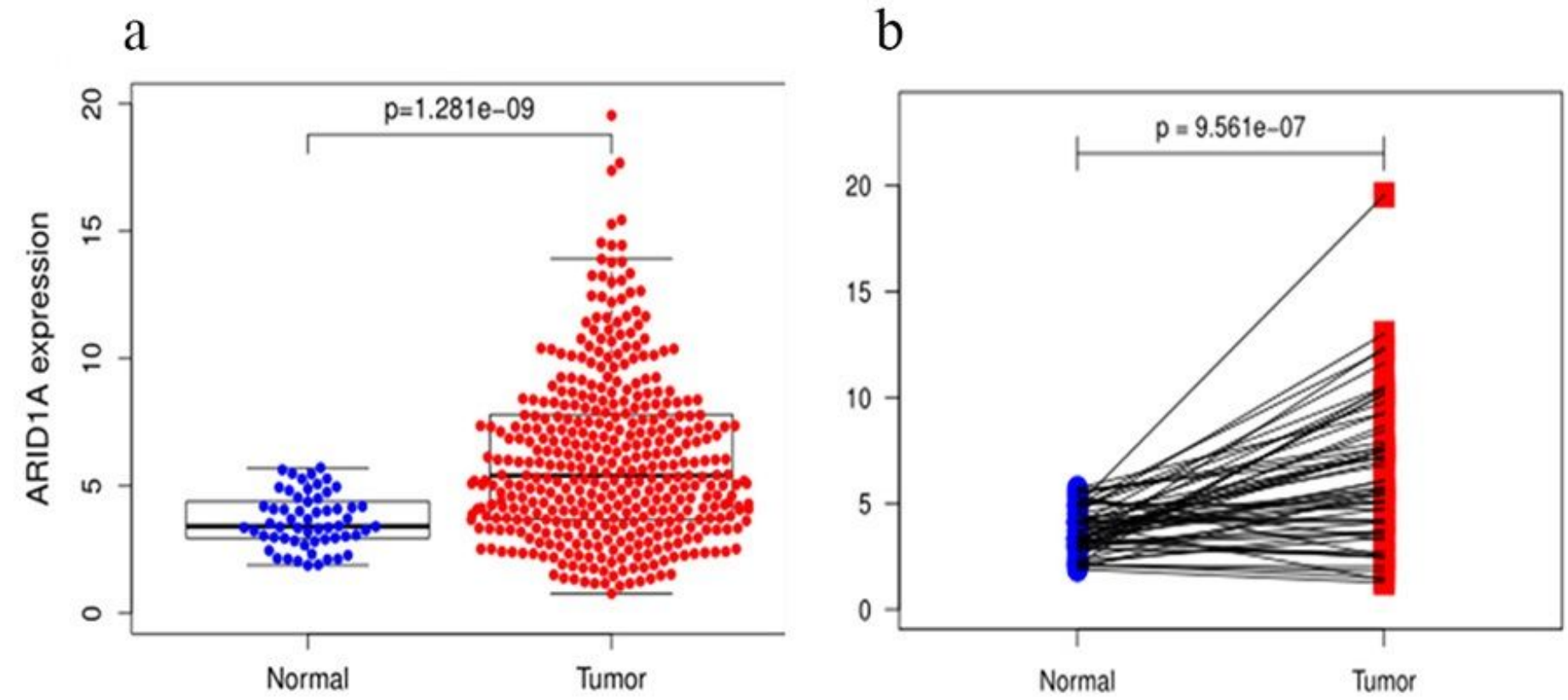

Figure 1

ARID1A expression in liver cancer tissues and normal tissues. (a) scatter plot (b) paired difference plot 

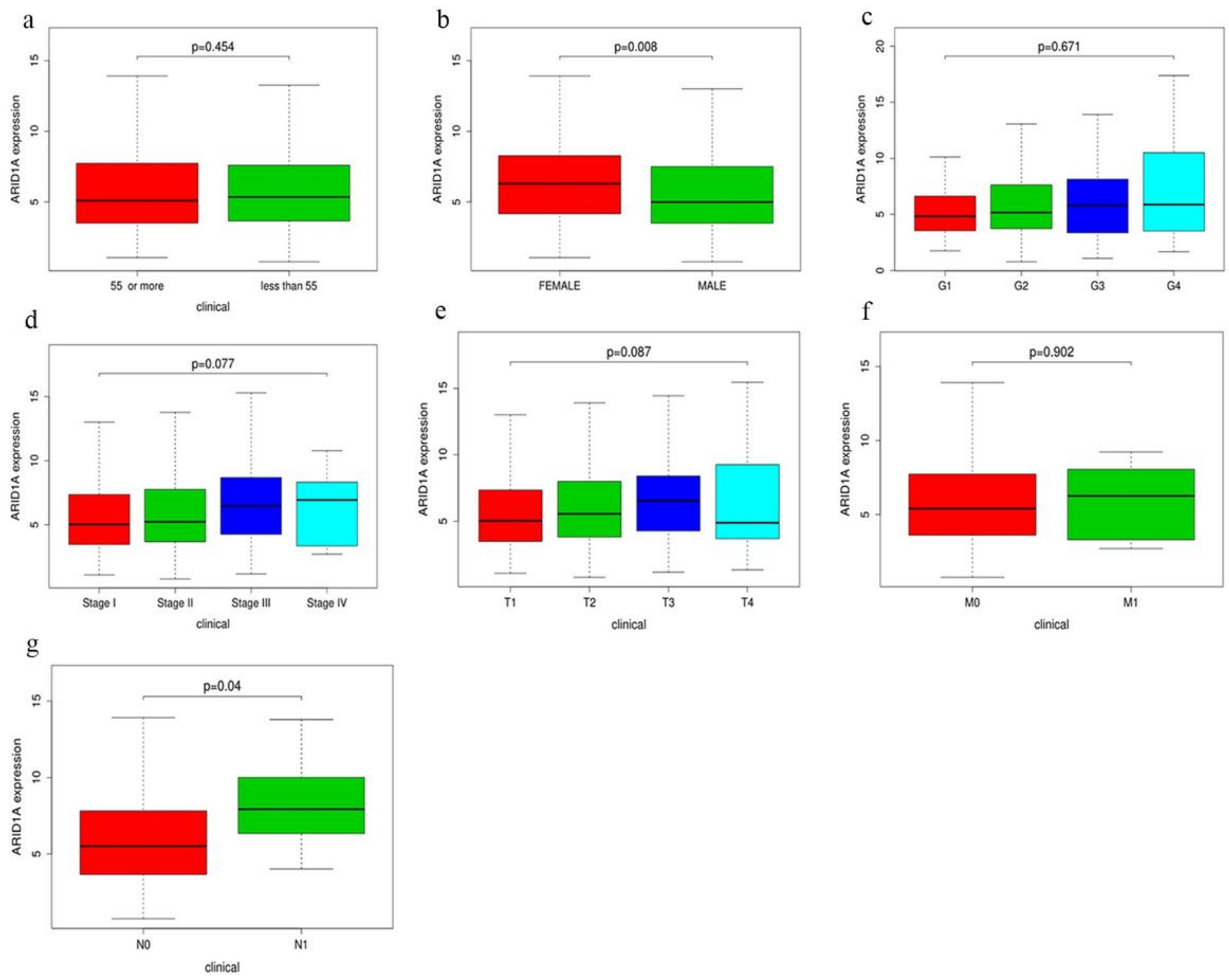

Figure 2

Association of ARID1A expression with clinical variables. a: age; b: gender; c: grade; d: stage; e: tumor topography; f: distant metastases; g: lymph node metastasis. 
$\operatorname{ARID1A}(p=0.038)$

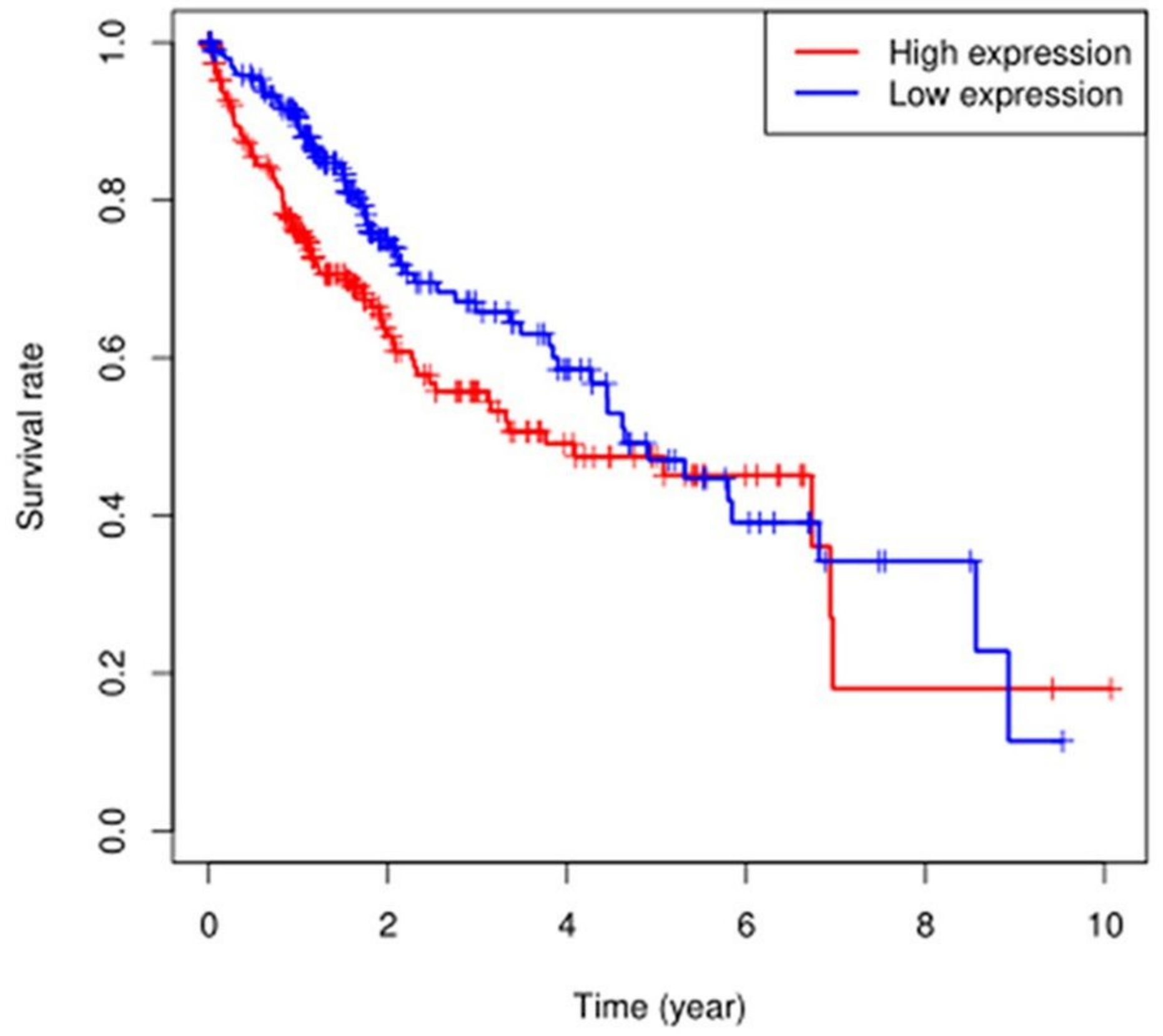

Figure 3

ARID1A expression and overall survival in liver cancer patients in TCGA 

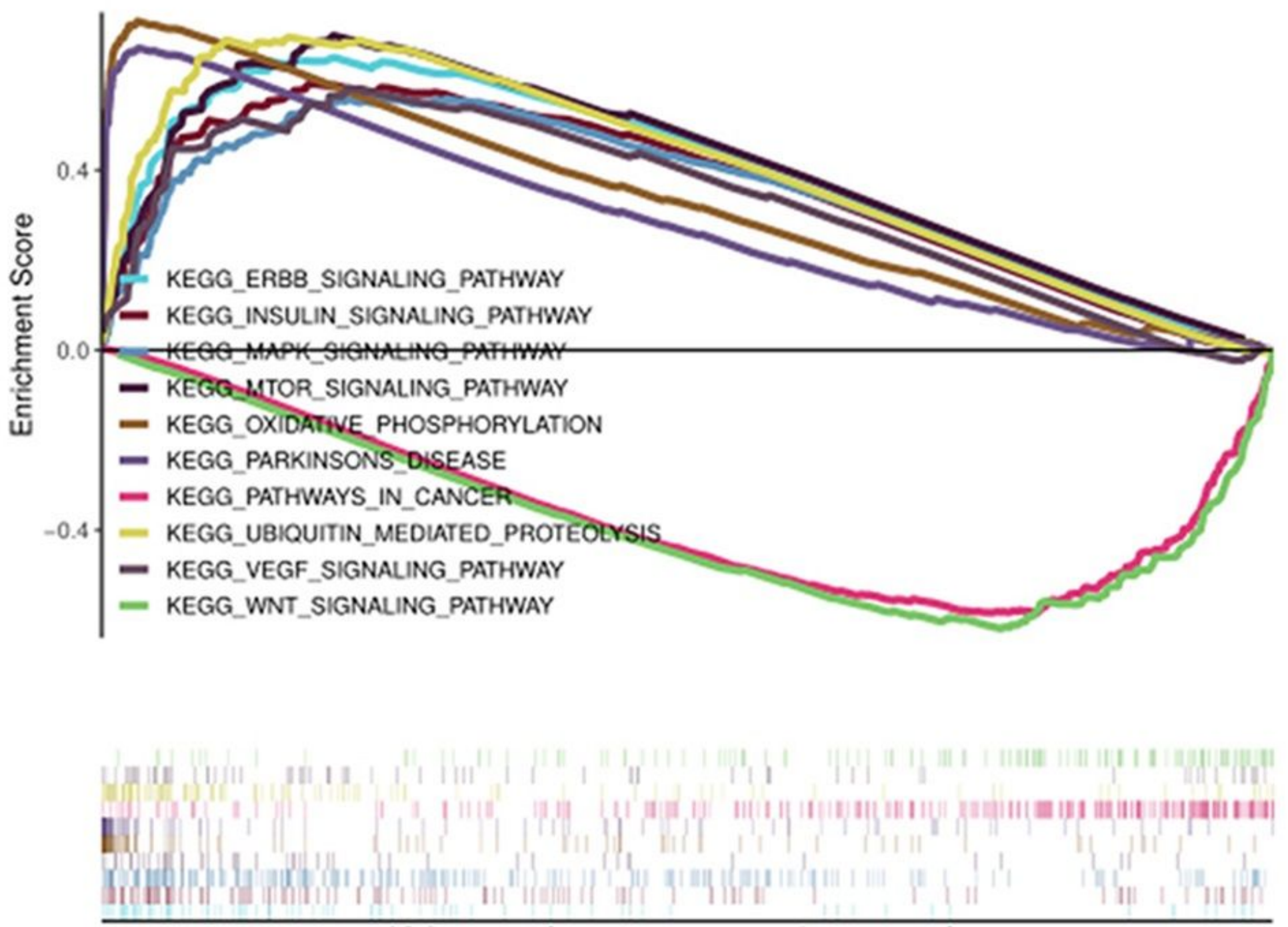

high expression<---------->low expression

Figure 4

Enrichment plots from gene set enrichment analysis (GSEA). 